\title{
HFRS with Severe Heart Liver and Renal Failure: a Case Report
}

\author{
Qing Zhou ${ }^{1}$, Meng-Hou Lu², Lei Fu ${ }^{2}$ and De-Ming Tan ${ }^{2}$
}

Hemorrhagic fever with renal syndrome (HFRS) is caused by hantavirus infection, which was characterized by abrupt high fever, systemic hemorrhage, hypotension and renal damage. Although multiple system organ damage was not uncommon, but multiple organ system failure were rare. Hereafter we report one case with simultaneous renal, heart and liver failure. In this case, we received some experience and lessons.

Key Words: HFRS; Heart failure; Liver failure; Renal failure; Hemodialysis

$\mathrm{H}$ emorrhagic fever with renal syndrome (HFRS) is caused by hantavirus infection, which was characterized by abrupt high fever, systemic hemorrhage, hypotension and renal damage. However, in addition to renal damage, multiple system organ damage was not uncommon. Yang reported that the rate of liver damage in patients with HFRS ranged from $56.5 \%$ to $81.4 \%$, and biopsy demonstrated hepatocytes degeneration or necrosis reached up to $97.6 \%$, and the presence of "hemorrhagic fever hepatitis" was universal; ${ }^{1}$ but multiple organ system failure were rare, hereafter we report one case with simultaneous renal, heart and liver failure.

\section{CASE REPORT}

A 24-year-old male peasant was admitted in the local county hospital due to 4 days abrupt fever, oliguria, skin and sclera jaundice. He had abrupt high fever which reached $40^{\circ} \mathrm{C} \quad 7$ days before and was associated with headache and orbital pain and persistent loin pain, and nausea and vomiting. He then received antibiotic therapy in the county hospital and his fever was resolved at the 3rd day; but symptoms deteriorated and hypotension occurred $(70 / 40 \mathrm{~mm} \mathrm{Hg})$, which although returned normal after resuscitation but followed by oliguria, with 24-hour urine volume as $300 \mathrm{ml}$ and deep colored looking like black tea, with progressively deepening skin jaundice and then he was admitted by our hospital through the emergence department being diagnosed as: HFRS complicated with acute liver failure -febrile shock and jaundice of uncertain reason.

\footnotetext{
${ }^{1}$ The Department of Infectious Disease, Zhuzhou Central Hospital, Zhuzhou 412000 ,China

${ }^{2}$ The Department of Infectious Disease, Central South University Xiangya Hospital, Key Laboratory of Viral Hepatitis,Changsha 410005, China

Correspondence: Meng-Hou Lu, E-mail: lumenghou@126.com
}

During the admission, his vital signs were $36.5^{\circ} \mathrm{C}$, pulse $78 / \mathrm{min}$, respiration: $22 / \mathrm{min}$ and BP $112 / 78 \mathrm{~mm}$ $\mathrm{Hg}$. He was conscious and clear, acutely ill, heavily yellow stained skin and sclera, multiple pinpoint reddish rashes on his face, neck, chest and both armpits without discoloration under pressure, also without ecchymosis; conjunctiva with mild edema, hyperemia and hemorrhage. There were rough breath sound in both sides of the chest, which were neither rhonchus nor rales. Mild swelled and tensed abdomen with tenderness and rebound pain occurred, with the right middle quadrant most prominent, and Murphy's sign was negative, but liver and spleen was not palpated, with shifting dullness and obvious percussion tenderness on both kidney areas. The laboratory test results of the patient was shown as Table 1 .

For this patient, indexes of hepatitis virus were negative, but the antibody against hantavirus was positive, and EB virus, cytomegalovirus and respiratory syncytial viral infections were excluded as well. Chest X-ray examination revealed pleurisy and effusion on both pleural cavities; abdominal ultrasonography demonstrated hepatosplenomegaly, ascitis $42 \mathrm{~mm}$; both kidneys revealed parenchymal lesion with grade $\mathrm{A}$.

This patient was diagnosed as HFRS within oliguria stage and managed according to restrict fluid intake and oral potassium supplementation, also furosemide was applied intravenously every 8 hours; isoglycyrrhrrhetic acid magnesium and meglumine adenosine cyclophosphate injections were taken to protect liver and heart functions; omeprazole and etamcylate were applied to protect from gastric acid and for hemostasis; Cefamandole sodium was taken to prevent from infection; intermittent infusion of frozen plasma supplement coagulation factors and albumin, various intensive supportive measures and aggressive 
Table 1. Laboratory test results of the patient

\begin{tabular}{llll}
\hline Laboratory test items & & Corresponding results & \\
\hline Blood routine & WBC $20.4 \times 10^{9} / \mathrm{L}$ & Neutrophils $58 \%$ & Platelets $41 \times 10^{9} / \mathrm{L}$ \\
Urinalysis & Occult blood $+/-$ & Protein +++ & RBC $+/ \mathrm{Hp}$ \\
Liver function & ALB $31 \mathrm{~g} / \mathrm{L}$ & TBil $158.7 \mu \mathrm{mol} / \mathrm{L}$ & DBil $81 \mu \mathrm{mol} / \mathrm{L}$ \\
& ALT $3538.9 \mathrm{U} / \mathrm{L}$ & AST $1228 \mathrm{U} / \mathrm{L}$ & \\
Renal function & BUN $33.7 \mathrm{mmol} / \mathrm{L}$ & $\mathrm{Cr} 785.2 \mu \mathrm{mol} / \mathrm{L}$ & $\mathrm{UA} 1101 \mu \mathrm{mol} / \mathrm{L}$ \\
Cardiac enzymology & LDH $1857.3 \mathrm{U} / \mathrm{L}$ & CK $432 \mathrm{U} / \mathrm{L}$ & CK-MB 31 U/L \\
Prothrombin time activity (PTA) & $35 \%$ & & \\
\hline
\end{tabular}

blood hemodialysis were also applied. With the above measures, the symptoms were alleviated, urine volume gradually increased and reached $2300 \mathrm{ml} / 24$ hours, and liver function and coagulation parameters and cardiac enzymology all returned to normal levels; but BUN $(15.4 \mathrm{mmol} / \mathrm{L})$ and $\mathrm{Cr}(926.5 \mu \mathrm{mol} / \mathrm{L})$ persisted abnormal, and the patient received additional hemodialysis. During the 15th hospitalized day, the patients urine volume of 24 hours reached $4700 \mathrm{ml}$, so late polyuric stage of the patient was considered, and the furosemide was suspended intravenously; then the patient developed to chest stuffiness, dyspnea, cough with whitish frothy sputum, and orthopnea with tachycardia and tachypnea at 11 PM during the next day and bilateral course rales could be heard through auscultation, with BP 170/110 $\mathrm{mm} \mathrm{Hg}$, heart rate 110 beats/min, respiration rate $27 / \mathrm{min}$ and ECG demonstrated nonspecific ST alterations indicating acute hypervolemic pulmonary edema which might be due to excessive reabsorption that resulted in fluid overload, so the patient maintained orthopnea position, supplementation of oxygen, furosemide $100 \mathrm{mg}$ intravenously and emergency hemodialysis, then his symptoms relieved 24 hours thereafter. Then the patient received furosemide $140 \mathrm{mg}$ intravenously every 8 hours, and urine volume increased steadily and reached the maximal as $5450 \mathrm{ml} / 24$ hours. His renal function returned normal, with BUN $6.8 \mathrm{mmol} / \mathrm{L}$, Cr 125.6 $\mu \mathrm{mol} / \mathrm{L}$ and UA $318 \mu \mathrm{mol} / \mathrm{L}$. Then he was discharged at the 29th hospitalized day.

\section{DISCUSSION}

HFRS is caused by viral infection characterized by systemic microvasculolymphatic, with the pathogenesis as renal and hepatic injuries and large amplitude fluctuations of circulatory volume that disordered homeostasis. And thus therapies should be aimed to manage the accelerated pathophysiologic alterations accordingly. In this case we could get the experiences and lessons as following:

Therapeutic measures should be aimed to maintain a stable homeostasis to improve microcirculation and protect heart, kidney and liver from injury: Wan et $\mathrm{al}^{2}$ reported 369 HFRS cases complicated with liver injuries and found that liver injury occurred mostly during the oiguric stage which accounted $77 \%$, and was correlated with the severity of the insults. The pathogenesis mechanisms include direct viral injury, liver microcirculatory disturbances, impaired blood infusion and immune injuries. ${ }^{3}$ During the early stage, hepatocytes invaded by virus occured cell structural damage and immune complex deposition which could induce injury, ${ }^{3}$ hypotensive and microcirculation disturbances during oliguric stage and thus ischemia and hypoxia were responsible to hepatocytes degeneration and necrosis. ${ }^{4,5}$ As to this case, his liver functional impairment occurred early and aggravated during the oliguric stage, with complicated reasons; however, even if the liver damage was severe, the recovery was rapid, so it was considered that in addition to the HFRS as a self-limited disease and the insult was transient, the maintainence of homeostasis improved liver microcirculation and liver protective therapeutic measures were all involved. It is suggested that early liver function impairment must never be neglected and proper management is significant in prevention of liver function failure.

Early hemodialysis to reduce fluid overload and heart failure is and important measure. Potential myocardial and conductive system damages in severe HFRS patients were the pathophysiologic basis for the development of arrhythmias, left ventricular systolic failure and sudden death. Kong et al observed 25 severe HFRS patients and found 20 patients with abnormal ECG, among whom 10 severe cases with elevated cardiac enzyme that were positively correlated with BUN and serum creatinine, which suggested that during acute renal injury stage, toxins accumulation also a threaten to heart injury. ${ }^{6}$ This patient occurred severe heart injury at a early stage, furthermore, he developed to PE when his diuretics therapy was suspended prematurely, so we experienced that his early multiple hemodialysis effectively reduced the fluid overload and improved the prognosis, as well as shock correction, accurate fluid infusion rate and the administration of cardiac protective remedies.

The diuretic therapy should not be suspended 
prematurely in critical HFRS patients: although the urine volume increased during the transition period from oliguric stage to polyuric stage, however, the renal function restoration was only initiated, while the retained large amount of excessive interstitial and serous cavity fluid during the late febrile stage and hypotensive shock stage infiltrating into extravascular space was reabsorbed to become intravascular volume, furthermore, the metabolic products increase indefinitely and continuously; premature suspendent of diuretic therapy resulted in the decrease of urine output which rendered the patient vulnerable to hypervolemic syndrome. So it is suggested that the diuretic therapy should not be prematurely suspended.

\section{REFERENCES}

1. Yang WS. Hemorrhagic fever with renal syndrome. Beijing: People's Medical Publishing House; 1999:185-186.

2. Wan BM, Li HL, Wang J, Wang HL, Guo Q. Implication of liver function in hemorrhagic fever patients with renal syndrome. Zhonghua Gan Zang Bing Za Zhi (Chin) 2005;13(5):387-388.

3. Zhang SF, Feng HF, Zhang JH. The clinical analysis about Hemorrhagic fever with renal syndrome with liver damage. Zhonghua Wu Zhen Xue Za Zhi (Chin) 2006;6(5):913-914.

4. Wei $\mathrm{L}, \mathrm{Yu} \mathrm{YZ}$. A1teration of plasma coagulation and fibrinolysis in epidemic hemorrhagic and its relation with liver damage. Zhonghua Chuan Ran Bing Za Zhi (Chin) 1995;13:21-23.

5. Saggio I, Gloaguen I, Poiana G, Laufer R. CNTF variants with increased biological potency and receptor selectivity define a functional site of receptor interaction. EMBO J 1995;14:3045-3054.

6. Kong L, Ren ZX, Hu YL, Pei X. Analysis of 25 heart damage patients with critically illed hemorrhagic fever with renal syndrome. Zhongguo Quan Ke Yi Xue (Chin) 2005;8(8):664-665. 